\title{
TLR9 ligand induces the generation of CD20+ plasmablasts and plasma cells from CD27+ memory B-cells
}

\section{Alexandrine Geffroy-Luseau ${ }^{1,2+}$, David Chiron ${ }^{1,2 \dagger}$, Géraldine Descamps ${ }^{1,2}$, Gaëtan Jégo ${ }^{1,2 \dagger}$, Martine Amiot ${ }^{1,2}$ and Catherine Pellat-Deceunynck ${ }^{1,2}{ }^{*}$}

1 INSERM, UMR_S892, Nantes, France

${ }^{2}$ UFR Médecine, Therapeutic Research Institute, Université de Nantes, Nantes, France

\section{Edited by:}

Isabelle Bekeredjian-Ding, University Hospital Heidelberg, Germany

Reviewed by:

To-Ha Thai, Beth Deaconess Israel Medical Center, USA

Dirk Alexander Mielenz, University of Erlangen-Nürnberg, Germany

${ }^{*}$ Correspondence:

Catherine Pellat-Deceunynck, INSERM, UMR_S892, Centre de Recherches en Cancérologie Nantes Angers, IRT-UN, 8, quai Moncousu, Nantes BP70721 F-44007, France.

e-mail: catherine.pellat-deceunynck@ inserm.fr

\section{${ }^{\dagger}$ Present address:}

Alexandrine Geffroy-Luseau, Atlantic Bone Screen, Nantes, France;

David Chiron, Weill Cornell Medical College, New York, NY, USA;

Gaëtan Jégo, University of Burgundy, INSERM, U866, 7 Boulevard Jeanne d'Arc, 21033 Dijon, France.
Plasma cells (PCs) have a heterogeneous phenotype in humans. While bone marrow PCs are CD20-CD138+, tonsil PCs are CD20+CD138+/- and peripheral plasmablasts (PBs) are CD20-CD138-. In vitro, PCs are mainly generated by the activation of CD27+ memory B-cells through transient stimulation of CD40, and their phenotype appears similar to that of bone marrow PCs. While CD20 expression is lost at the plasmablastic stage, CD138 expression appears only at the PC stage. Thus, the CD20+CD138 \pm phenotype of tonsil PCs does not represent an intermediate stage in the differentiation of memory Bcells into PCs. Because it has been previously shown that TLR9 activation was more able than CD40 stimulation to induce the differentiation of IgM+CD27+ B-cells, we wondered whether TLR9 or CD40 stimulation would induce the same phenotype of PCs. Thus, we compared the differentiation of CD27+ B-cells isolated from either the tonsils or peripheral blood and stimulated with either CD40L-expressing fibroblasts or a TLR9 ligand, CpG oligodeoxynucleotide (CpG ODN). We observed that CpG ODN mainly induced CD27+ Bcell differentiation into CD20+CD38+CD138- PBs and CD20+CD38+CD138 \pm PCs, which appear similar to tonsil PCs. Removal of CpG ODN during differentiation induced a decrease in the CD20+ plasmablastic population, and, conversely, stimulation of CD40L-induced preplasmablasts with CpG ODN increased the population of CD20+CD38+ PBs. Analysis of Ig secretion showed that CpG ODN induced increased IgM secretion compared to CD40L. PCs from patients with multiple myeloma, the malignant counterpart of bone marrow PCs, rarely express CD20. We show that CpG ODN did not induce or increase CD20 in nine IgG or IgA myeloma cell lines. These data strongly suggest that $\mathrm{CpG}$ ODN mainly targets CD27+ lgM+ B-cells.

Keywords: plasma cells, survival, CpG, CD20

\section{INTRODUCTION}

Plasma cells (PCs) are terminally differentiated B-cells (ShapiroShelef and Calame, 2005; De Vos et al., 2006; Moser et al., 2006; Radbruch et al., 2006). Memory B-cells are activated in secondary immune organs and differentiate into plasmablasts (PBs), which migrate to inflamed tissues, lamina propria, and bone marrow, where they differentiate into PCs (Arpin et al., 1997). Long-lived PCs that are the source of protective antibodies are mainly located within the bone marrow. PCs are found not only within bone marrow but are also located within secondary immune organs such as the spleen, lymph nodes, and tonsils. PCs in all organs exhibit a shared phenotype, but some differences can be observed. For example, tonsil PCs are $\mathrm{CD} 20+$ and $\mathrm{CD} 138+/-$, in contrast to BM PCs that are CD20- but CD138++ (Pellat-Deceunynck et al., 1994; Medina et al., 2002; Robillard et al., 2005). During B-cell differentiation into PCs, CD20 expression is lost at the

Abbreviations: MFI, mean fluorescence intensity; PB, plasmablast; PC, plasma cell; prePB, pre-plasmablast. plasmablastic stage and CD138 upregulation occurs at the PC stage (Jego et al., 2001). In vivo, expansions of non-malignant polyclonal PBs and PCs are transiently observed in patients with IL-6 producing tumors or in the context of acute infections (Jego et al., 1999). These PBs and PCs never express CD20 (Jego et al., 1999). PCs from tonsil have been considered to be immature PCs, which can leave the tonsil to migrate to the bone marrow. However, phenotypic studies of cytoplasmic Ig+ (cIg+) cells in the blood during a secondary immune response have shown that cIg+ cells have a phenotype typical for PBs, CD20-CD138-, making it unlikely that tonsil PCs are the precursors to BM PCs or peripheral PBs (Medina et al., 2002; Odendahl et al., 2005; Robillard et al., 2005). Based on phenotype, the tonsil PCs appear to be different from other types of PCs or PBs.

In vitro, $\mathrm{PBs}$ and $\mathrm{PCs}$ have been mainly obtained from memory B-cells activated by CD40L (Arpin et al., 1995; Jego et al., 2001). In this culture system, the PBs and PCs obtained never expressed CD20. Microbial products activate B-cells, which express a wide range of toll-like receptors (TLRs). Indeed, B-cells express TLR9 and are strongly activated by TLR9 ligand, CpG 
oligodeoxynucleotides (CpG ODN). Bernasconi et al. (2002) have reported that $\mathrm{CpG}$ ODN induce the proliferation and differentiation of CD27+ B-cells into PCs and that CpG ODN, in contrast to CD40L, strongly stimulate IgM+ B-cells. Thus, we wondered whether the activation of CD27+ B-cells by two very different culture systems (CD40L or CpG ODN) would generate PBs and PCs with similar or different features. Our data show that CpG ODN, in contrast to CD40L, promote the generation of CD20+ PCs.

\section{MATERIALS AND METHODS ANTIBODIES AND REAGENTS}

Anti-CD20-FITC, anti-CD38-APC, and IgG1-APC control were purchased from BD Biosciences (Le Pont de Claix, France). Anti-CD45-FITC, anti-CD138-PE, IgG1-FITC control, and IgG1PE control were purchased from Beckman Coulter (Roissy, France). Blocking anti-CD40L mAb was purchased from Alexis (Coger, Paris, France). The recombinant human cytokines IL2, IL10, and IL15 were obtained from R\&D (Minneapolis, MN, USA). The TLR9 ligand, CpG phosphorothioated ODN (50TCGTCGTTTTGTCGTTTTGTCGTT-30, CpG2006), was purchased from InvivoGen (San Diego, CA, USA).

Antibodies against $\mathrm{Bim}(\mathrm{Abs})$ and actin $(\mathrm{mAb})$ were purchased from Millipore (Merck Serono, Lyon, France), anti-Mcl-1 Abs and anti-caspase $3 \mathrm{mAb}$ were purchased from Santa Cruz Biotechnology (Tebu-Bio, Le Perray en Yvelines, France), anti-Bcl-2 mAb was purchased from Dako (Trappes, France), anti-p27 and anti-PCNA mAbs were purchased from BD Pharmingen (BD Biosciences, Le Pont de Claix, France), anti-p18 mAb was purchased from Cell Signaling Technology (Ozyme, Saint-Quentin en Yvelines, France), anti-Bcl-xL Abs were purchased from Transduction Laboratories (BD Biosciences, Le Pont de Claix, France) and anti-survivin Abs were purchased from R\&D Systems Europe (Abingdon, UK).

\section{GENERATION OF PLASMABLASTS AND PLASMA CELLS}

Human cells were obtained from normal donors after informed consent. Tonsils were obtained from children and teenagers undergoing routine tonsillectomy. Following isolation by FicollHypaque centrifugation and adherence, the lymphocytes were cryopreserved. After thawing, the T cells were removed by sheep rosetting (two rounds), and CD27+ B-cells were sorted (MACS, Miltenyi Biotec, Germany), as previously described (Jego et al., 2001; Geffroy-Luseau et al., 2008). Peripheral blood samples from donor volunteers were provided by the French Blood Institute (Etablissement Français du Sang, Nantes). After Ficoll-Hypaque centrifugation and adherence, CD19+CD27+ B-cells were sorted with the CD19 Multisort Kit (MACS, Miltenyi).

Generation of PBs and PCs by transient activation of CD40 was performed as initially described by Arpin et al. (1995), though slightly modified (Arpin et al., 1997; Jego et al., 2001; GeffroyLuseau et al., 2008). Briefly, CD27+ B-cells were incubated for 3 days (Day 3) over mitomycin-C-treated ( $45 \mathrm{~min}, 75 \mu \mathrm{g} / \mathrm{ml}$ ) CD40L-expressing fibroblasts (a kind gift of Dr. T. Defrance, Lyon, France) in the presence of $50 \mathrm{U} / \mathrm{ml} \mathrm{IL2}$ and $100 \mathrm{ng} / \mathrm{ml} \mathrm{IL10.}$ Thereafter, the cells were cultured for 4 (Day 7) to 7 days (Day 10) without fibroblasts, in the presence of IL2 $(50 \mathrm{U} / \mathrm{ml})$, IL10 (100 ng/ml), and blocking anti-CD40L mAb $(2 \mu \mathrm{g} / \mathrm{ml})$.
Generation of PBs and PCs by CpG2006 treatment in the presence of IL15 or IL2 plus IL10 was performed as previously described by Bernasconi et al. (2002). Briefly, CD27+ B-cells were cultured for up to 10 days with $3 \mu \mathrm{g} / \mathrm{ml} \mathrm{CpG} \mathrm{ODN} 2006$ plus either $100 \mathrm{ng} / \mathrm{ml}$ IL15 or $50 \mathrm{U} / \mathrm{ml}$ IL2 and $100 \mathrm{ng} / \mathrm{ml}$ IL10. Proliferation was monitored by PI staining.

\section{IDENTIFICATION OF PLASMABLASTS AND PLASMA CELLS}

Plasmablasts and PCs were identified by CD20, CD38, and CD138 expression with a FACSCalibur flow cytometer (Becton Dickinson), as previously described (Jego et al., 1999, 2001; Geffroy-Luseau et al., 2008).

\section{ISOLATION OF prePBs}

Isolation of pre-plasmablast (prePBs) was performed as described previously (Geffroy-Luseau et al., 2008). Briefly, CD27 B-cells were cultured for 3 days with CD40L-expressing fibroblasts in the presence of IL2 plus IL10 and then for 2 days with IL2 plus IL10 in the presence of anti-CD40L mAb. At this time, the culture contained prePBs (CD20+/-) and remaining B-cells (CD20++). To obtain a pure population of prePBs engaged in differentiation, the B-cells were removed with CD20 immunomagnetic beads by using a positive selection column (Column MS, Miltenyi), which selects only highly labeled cells.

\section{Ig SECRETION}

IgG, IgA, and IgM secretion was determined by ELISA, as previously described (Geffroy-Luseau et al., 2008).

\section{WESTERN BLOTTING}

Cells were pelleted and resuspended in lysis buffer $(10 \mathrm{mM}$ Tris$\mathrm{HCl} \mathrm{pH}=7.6,150 \mathrm{mM} \mathrm{NaCl}, 5 \mathrm{mM}$ EDTA, $1 \mathrm{mM}$ PMSF, $2 \mu \mathrm{g} / \mathrm{ml}$ aprotinin, $1 \%$ Triton X100). After $40 \mathrm{~min}$ on ice, lysates were cleared by centrifugation at $10,000 \times g$ for $30 \mathrm{~min}$ at $4^{\circ} \mathrm{C}$. Protein concentration was measured using bicinchoninic acid (BCA Protein assay, Pierce, Rockford, IL, USA). Cleared lysates $(70 \mu \mathrm{g})$ were separated by SDS-PAGE (12.5 or 15\% acrylamide) and electrotransferred to polyvinylidene difluoride membranes. Western blot analysis was performed by standard techniques with ECL detection (Pierce Perbio Science France, Brebières, France).

\section{STIMULATION OF MYELOMA CELLS}

Human myeloma cell lines (HMCLs) have been previously described (Moreaux et al., 2011). Cells $(500,000 / \mathrm{ml})$ were cultured for 2 days with or without CpG $2006(5 \mu \mathrm{g} / \mathrm{ml})$ in the presence of or without IL15 (100 ng/ml), as described previously (Jego et al., 2006; Chiron et al., 2009). After culture, cells were stained with anti-CD20-FITC or IgG1-FITC control mAb, and the fluorescence was analyzed with a FACsCalibur flow cytometer (Becton Dickinson).

\section{RESULTS \\ PBs AND PCs GENERATED IN THE PRESENCE OF CpG ODN REMAIN CD20+}

Because tonsil and bone marrow PCs display very different phenotypes (CD20, CD138, Ig isotype), we wondered whether polyclonal activation of memory B-cells through different mechanisms, 
CD40L stimulation versus CpG ODN stimulation, could promote the generation of phenotypically different PCs. To answer this question, CD27+ B-cells were purified from tonsils and activated in the presence of IL2 and IL10 with either CD40L-expressing 3T6 fibroblasts or with CpG2006. Because continuous activation of Bcells by CD40L prevented differentiation (Figure 1A; Arpin et al., 1995), we performed a transient coculture (3 days) followed by a 4to 7-day culture in the presence of anti-CD40L-blocking mAbs to neutralize any remaining CD40L+ cells. In contrast to the CD40L culture conditions, for CpG stimulation, the ligand was added at Day 0 with no washes to remove CpG ODN. At Day 7, both cultures displayed CD38+ PBs, confirmed by morphology, but CpG ODN-derived PBs were CD20+, albeit with diminished expression compared with the remaining CD20+CD38- B-cells, while CD40L-derived PBs were markedly CD20- (Figure 1B). Of note, at Day 10, both CD20+ and CD20+/- PCs expressed CD138, the marker of PCs (Pellat-Deceunynck et al., 1994). However, the level of CD138 expression was lower in CpG ODN-derived PCs than in CD40L-derived PCs. We wondered whether these differences in phenotype could be related to the presence of germinal center B-cells, as CD27 is expressed by both germinal center B-cells $(\mathrm{CD} 27+\mathrm{CD} 38+)$ and memory B-cells (CD38-CD27+).Thus, we purified CD27+ B-cells from the peripheral blood of healthy donors, which only contains memory B-cells and not germinal center B-cells. As shown in Figure 1C, peripheral CD27+ B-cells also generated CD20+ PBs when activated with CpG ODN, showing that this feature was not limited to tonsil CD27+ B-cells. To quantify the level of expression of CD20 in both cultures at Day 7 , we calculated the ratio of the levels of CD20 MFI of CD38+ cells (region 1) divided by the levels of CD20 MFI of CD38cells (region 2; four different tonsil samples and one peripheral blood sample). The intensity of CD20 in the CD40L cultures was of $0.0305 \pm 0.0157$, thus 3.6-fold lower compared with that of CpG cultures, $0.1106 \pm 0.0248(n=5, p=0.0018$, paired $t$-test; Figure 1C right). Finally, CpG ODN-derived PCs displayed a phenotype similar to that of in situ tonsil PCs, which are CD20+ CD138+/- (Medina et al., 2002; Robillard et al., 2005). Cell cycle analysis during the courses of differentiation did not show any significant differences between CD40L- and CpG ODN-generated PBs (Figure 1D). In both cases, proliferation decreased similarly during differentiation, and the incidence of subG1 peak increased similarly to reach $45.7 \pm 10.59$ and $45 \pm 12.70 \%$ of total cells, in the CD40L and CpG culture, respectively $(n=3, p=$ NS paired $t$-test). Because IL15 has been shown to be very efficient at promoting memory B-cell differentiation in the presence of CpG ODN (Bernasconi et al., 2002), we wondered whether it could be more or less efficient than IL2 + IL10 in supporting the generation of CD20+ PBs. As shown in Figure 1E, CpG ODN-generated PBs (from peripheral or tonsil B-cells) showed similar levels of CD20 expression in the presence of IL2 and IL10 as they did in the presence of IL15.

\section{REMOVAL OR ADDITION OF CpG ODN DURING DIFFERENTIATION MODULATED CD2O EXPRESSION}

We next wondered whether CpG ODN could directly modulate CD20 expression. To test this, we remove CpG ODN from CpG ODN-stimulated B-cells on day 3. As shown in Figure 2A, removal of CpG ODN slightly increased the proportion of cells with lower CD20 from $46 \pm 5$ to $64 \pm 7 \%$, compared with $65 \pm 12 \%$ for the CD40L culture. The CD20 intensity was slightly decreased too from $0.094 \pm 0.010$ to $0.055 \pm 0.010(n=3, p=0.026)$, but it still remained around twofold higher than that of CD40Ldriven PCs $(0.030 \pm 0.010, p=0.085)$. Reciprocally, we added CpG ODN to purified prePBs engaged in the differentiation process after transient CD40 activation. The remaining CD20+ B-cells were depleted 2 days after CD40L removal (Day 5) in order to target only prePBs and to exclude any recruitment of "resting" CD20+ B-cells (Geffroy-Luseau et al., 2008). As shown in Figure $2 \mathbf{B}$, the addition of $\mathrm{CpG}$ ODN slightly increased the proportion of CD38+CD20+ cells from $3.9 \pm 0.27$ to $15.25 \pm 1.51 \%$ at day 10, $n=3, p=0.0015$ (Figure 2B right, upper panel). In the CpG culture, CD20+CD38+ cells were only partly CD138+, with $7 \%$ of cells being CD20+CD138+ and $12 \%$ of cells being CD20+CD138-, compared with 2 and $2 \%$ in the culture without $\mathrm{CpG}$, respectively (illustrated in Figure 2B left). The proportion of $\mathrm{CD} 138+\mathrm{CD} 20+$ cells was higher in the culture with $\mathrm{CpG}$, $7.2 \pm 0.7 \%$, compared with the culture without $\mathrm{CpG}, 3.6 \pm 0.9 \%$, $p=0.073$ (Figure 2B right, lower panel). Thus, although $\mathrm{CpG}$ increased the proportion of CD20+ PBs and PCs, it did not prevent the appearance of CD20- PBs and CD20- PCs. These results suggest that $\mathrm{CPG} O \mathrm{ODN}$, in contrast to $\mathrm{CD} 40 \mathrm{~L}$, increased the survival of CD20+ PBs.

\section{MODULATION OF PROLIFERATION AND SURVIVAL PROTEINS IS SIMILAR IN CD40L- AND CpG ODN-GENERATED PBs AND PCs}

$B$-cell differentiation requires the concomitant regulation of both survival and proliferation (Chen-Kiang, 2003; Vikstrom and Tarlinton, 2011). Among all Bcl-2 family molecules, Bim, Mcl-1, and $\mathrm{Bcl}-2$ are three major regulators of B-cell and PC survival (Puthier et al., 1999; Vikstrom and Tarlinton, 2011). CDK inhibitors, especially p18, are required for PC generation (Chen-Kiang, 2003). We wondered whether CD40L and CpG ODN differentially modulated the expression of these molecules. Tonsil CD27+ B-cells (Day 0) were cultured either with IL2/IL10/CpG ODN for 7 days or with IL2/IL10/CD40L for 3 days (Day 3) followed by 2 days with IL2/IL10, after which CD20+/- prePBs and CD20+ B-cells were purified (Day 5). PrePBs were cultured for an additional 2 days in the presence of IL2 and IL10 (Day 7). Cells were then collected and lysed, and protein expression was analyzed by western blot at the indicated times (Figure 3). Upon CD40L or CpG ODN stimulation, expression of all three isoforms of Bim, Mcl1 full length (FL), Bcl-xL, PCNA, and survivin increased, while the expression of Bcl-2, p27 (Kip1), and p18 (INK4c) decreased (Day 3). Modulation of Bcl-2, PCNA, p27, and p18 was in agreement with the proliferation increase. Once CD27+ B-cells entered cell cycle induced by either CD40L or CpG, they switched from a $\mathrm{Bcl}-2$ expression toward a Mcl-1 and $\mathrm{Bcl}-\mathrm{xL}$ expression suggesting that cycling $\mathrm{CD} 27+\mathrm{B}$-cells should be rather Mcl-1/Bim or $\mathrm{Bcl}-\mathrm{xL} / \mathrm{Bim}$ than $\mathrm{Bcl}-2 /$ Bim primed for death. During subsequent differentiation, Bim expression remained high, Mcl-1 FL expression decreased, levels of the pro-apoptotic Mcl-1 cleaved form C1 increased (Ménoret et al., 2010), Bcl-xL expression remained high while Bcl-2 expression remained low. Although Bcl-2 expression was stronger in $\mathrm{CPG} O \mathrm{ODN}$-stimulated $\mathrm{PBs} / \mathrm{PCs}$ than in 

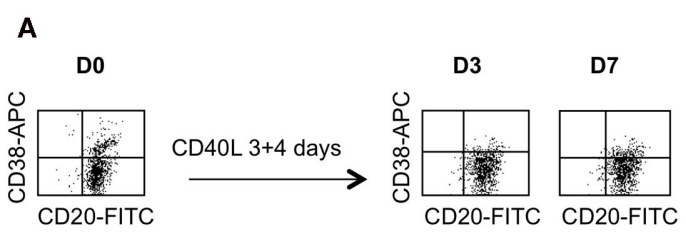

B

D3

D7

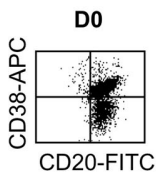
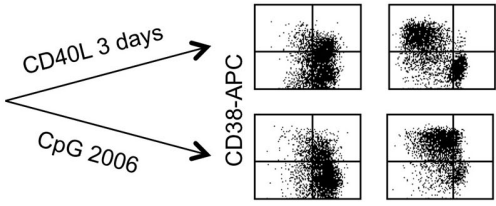

CD20-FITC
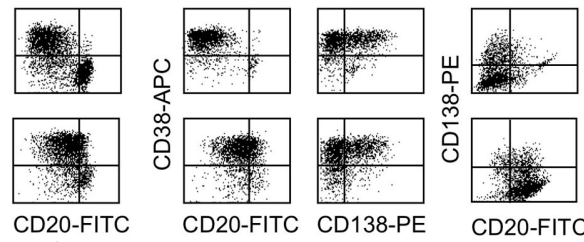

CD20-FITC

CD138-PE

CD20-FITC

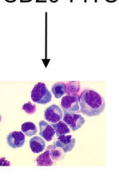

C
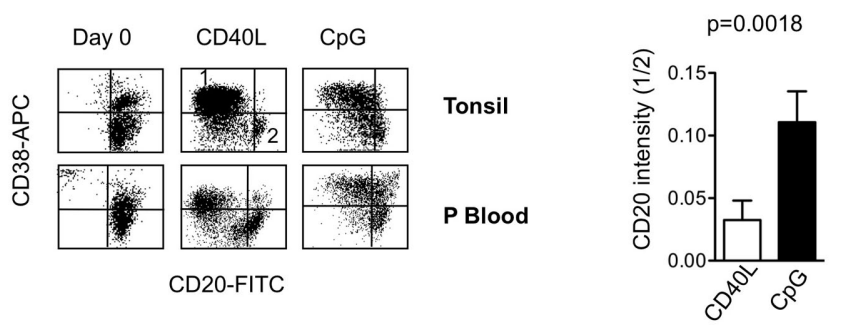

D

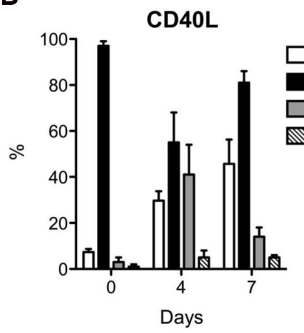

CpG

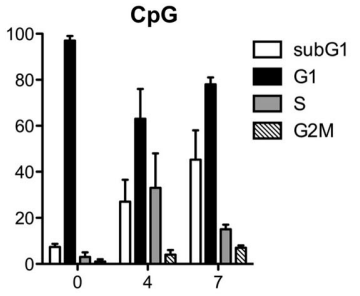

E

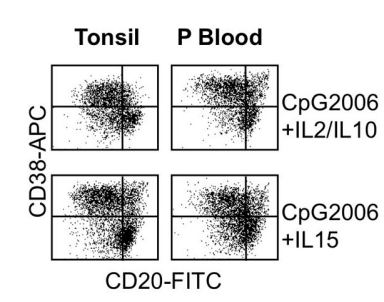

FIGURE 1 | (A) Continuous stimulation of CD27+ B-cells with CD40L-expressing fibroblasts prevented PC generation. CD27+ B-cells were purified from the tonsils of healthy donors, as described previously (Geffroy-Luseau et al., 2008 and Materials and Methods). B-cells ( 800,000 cells $/ \mathrm{ml}$ ) were cultured for 3 days with CD40L-expressing fibroblasts in the presence of IL2 and IL10 and harvested and cultured again for 4 days with CD40L-expressing fibroblasts in the presence of IL2 and IL10. The phenotype of the cells (CD20, CD38) was assessed by double staining. Representative experiment out of three. (B) Comparison of PCs generated from CD27+ B-cells either activated with CD40L-expressing fibroblasts or cultured in the presence of CpG2006. B-cells $(800,000$ cells $/ \mathrm{ml})$ from the same donor were either cultured for 3 days with CD40L-expressing fibroblasts and then cultured for 4 (Day 7) to 7 (Day 10) days with IL2 plus IL10 in the presence of blocking anti-CD40L mAb or cultured for 7-10 days in the continuous presence of CpG2006 (3 $\mu \mathrm{g} / \mathrm{ml})$ and IL2 plus IL10. The phenotype of the cells (CD20, CD38, CD138) was assessed by double or triple staining as indicated in the figure. May-Grünwald Giemsa staining of cells cultured for 7 days with CpG ODN plus IL2 and IL10 showed a typical PB morphology (eccentrically shaped nucleus and relatively abundant basophilic cytoplasm with archoplasm). The data represent the result of a representative experiment performed with one tonsil (three others were performed with three other independent donors). (C) Comparison of PCs generated from tonsil or peripheral blood CD27+ B-cells cultured in the presence of $\mathrm{CpG} 2006$. CD27+ B-cells (D0) were cultured as described in (B), and their phenotypes were analyzed at Day 7. Region 1 delineates CD38+CD20dim/- PBs, and region 2 delineates CD38-CD20+ B-cells. Right: the relative $\mathrm{CD} 20$ intensity in PBs at Day 7 was expressed as the ratio representative of $\mathrm{CD} 20 \mathrm{MFI}$ in $\mathrm{CD} 38+\mathrm{PBs}$ (region 1) divided by the $\mathrm{CD} 20 \mathrm{MFI}$ of the remaining B-cells CD20+CD38- (region 2). Five independent experiments were performed with $\mathrm{CD} 27+\mathrm{B}$-cells purified from four tonsils and one peripheral blood sample. (D) Cell cycle analysis of the differentiation of CD27+ B-cells cultured as described in (B). Histograms represent the proportion of cells in the subG1, G1, S, and G2M phases. SubG1 proportion was defined on the whole population. For $\mathrm{G} 1, \mathrm{~S}$, and G2M phases, the proportion was defined after exclusion of subG1 peak (Modfit). The data represent the mean \pm SD of three independent experiments performed with three independent donors. (E) Comparison of $\mathrm{CD} 20+\mathrm{PB}$ generation after $\mathrm{CpG} 2006$ stimulation in the presence of IL2 plus IL10 or IL15. CD27+ B-cells (tonsil or peripheral blood) were cultured as described in the legend of Figure 1B, with IL2 plus IL10 or with IL15. The phenotypes of the PBs were analyzed at Day 7. 


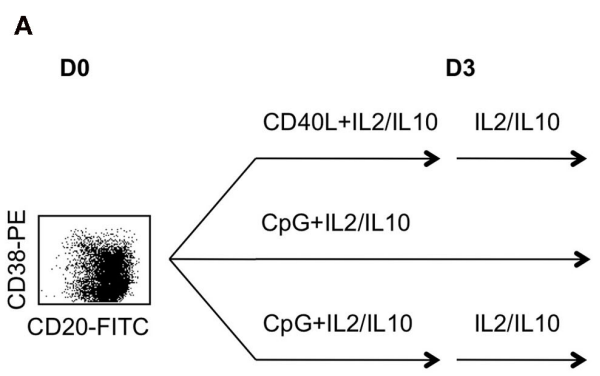

B prePBs

D5

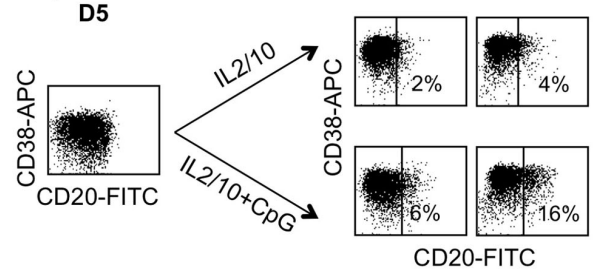

D7
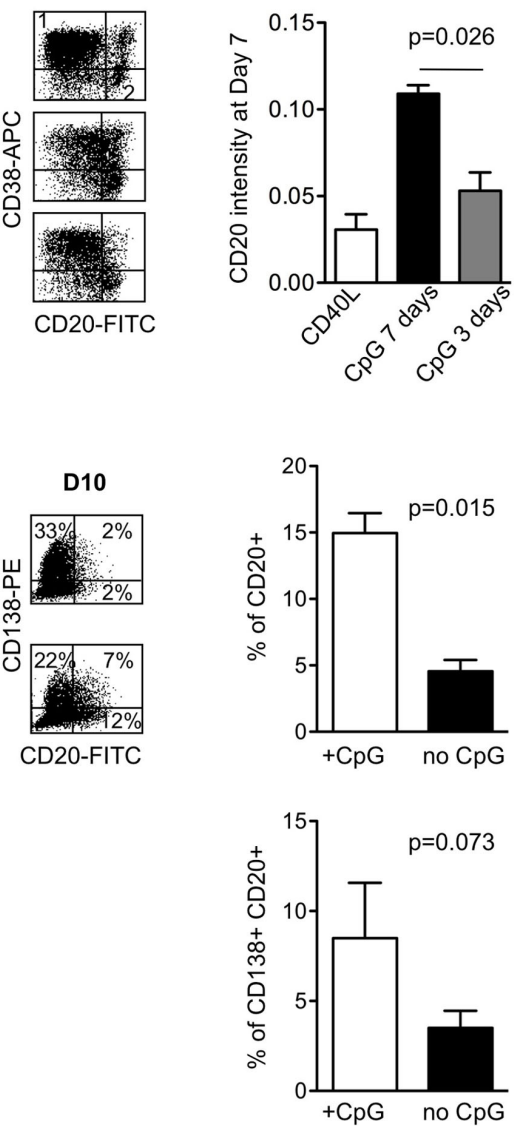

FIGURE 2 | (A) Removal of CpG ODN decreased the appearance of CD20+ PBs. Left: CD27+ B-cells from a tonsil were cultured with IL2 plus IL10 in the CD40L model (upper panel), with CpG2006 for 7 days (middle panel) or with $\mathrm{CpG} 2006$ for 4 days and then washed and cultured for 3 days without $\mathrm{CpG}$ (lower panel). The phenotype was analyzed at Day 7. The intensity of CD20 on PBs was expressed as the ratio ( $r$ ) of MFI staining on CD38+ cells (region 1) divided by CD38- cells (region 2). Representative experiment out of 3 . Right: data represent the mean $\pm S D$ of three independent experiments performed with three independent donors. (B) Addition of CpG ODN to CD40L-activated B-cells increased the appearance of $\mathrm{CD} 20+\mathrm{PBs}$. CD27+ B-cells from three independent

\begin{abstract}
donors were cultured for 3 days with CD40L-expressing fibroblasts in the presence of IL2 plus IL 10 and then for 2 days with IL2 plus IL10 in the presence of anti-CD40L mAb. The remaining $\mathrm{CD} 20++\mathrm{B}$-cells were removed to obtain a pure population of prePB cells engaged in differentiation (see Materials and Methods). PrePBs (Day 5) were then cultured in the presence of IL2 plus IL10 with or without CpG2006. The CD20 phenotype of PBs and PCs was analyzed 2 (Day 7) to 5 (Day

10) days after in double or triple staining. Representative experiment out of three. Right: data represent the proportion at Day 10 of CD20+ (upper panel) and of $\mathrm{CD} 20+\mathrm{CD} 138+$ (lower panel) in the culture with or without $\mathrm{CpG}$ (data represent the mean $\pm \mathrm{SD}$ of three independent experiments).
\end{abstract}

CD20-/CD40L-stimulated cells, no conclusion could be reached because the CpG ODN culture still contained B-cells, while the CD40L culture contained only PBs/PCs. Survivin and PCNA displayed regulation similar to that of $\mathrm{Mcl}-1$, increasing up to Day 5 and then decreasing. At Days 6 and 7,p27 expression remained low compared with unstimulated B-cells, but p18 expression was strongly increased, consistent with previous reports regarding expression of these factors during growth arrest and PC generation (Chen-Kiang, 2003). At Days 6 and 7, the decreased expression of anti-apoptotic proteins $\mathrm{Mcl}-1$ and $\mathrm{Bcl}-2$ and the increased levels of the Mcl-1-C1 form were in agreement with the high incidence of apoptosis in these cultures, as indicated by the strong cleavage of caspase 3 (Jego et al., 1999, 2001). In summary, CD40L- or CpG ODN-generated $\mathrm{PBs} / \mathrm{PCs}$ displayed a similar expression pattern of molecules involved in both the survival/apoptotic and cell cycle pathways.

\section{Cpg ODN INCREASED THE SECRETION OF IgM}

Bernasconi et al. (2002) have compared the proliferation and differentiation of switched (IgG, IgA) and non-switched (IgM) memory B-cells in response to either CpG ODN or bystander Tcell help. They showed that bystander T-cell help preferentially induced the proliferation of switched B-cells, while CpG ODN preferentially induced the proliferation of non-switched B-cells. Because the differentiation of B-cells into PCs is linked with proliferation, we theorized that $\mathrm{CpG}$ ODN should favor IgM secretion, while CD40L should favor secretion of IgG and IgA. Thus, we measured Ig secretion in the IL2/IL10 CD40L and CpG ODN/IL15 


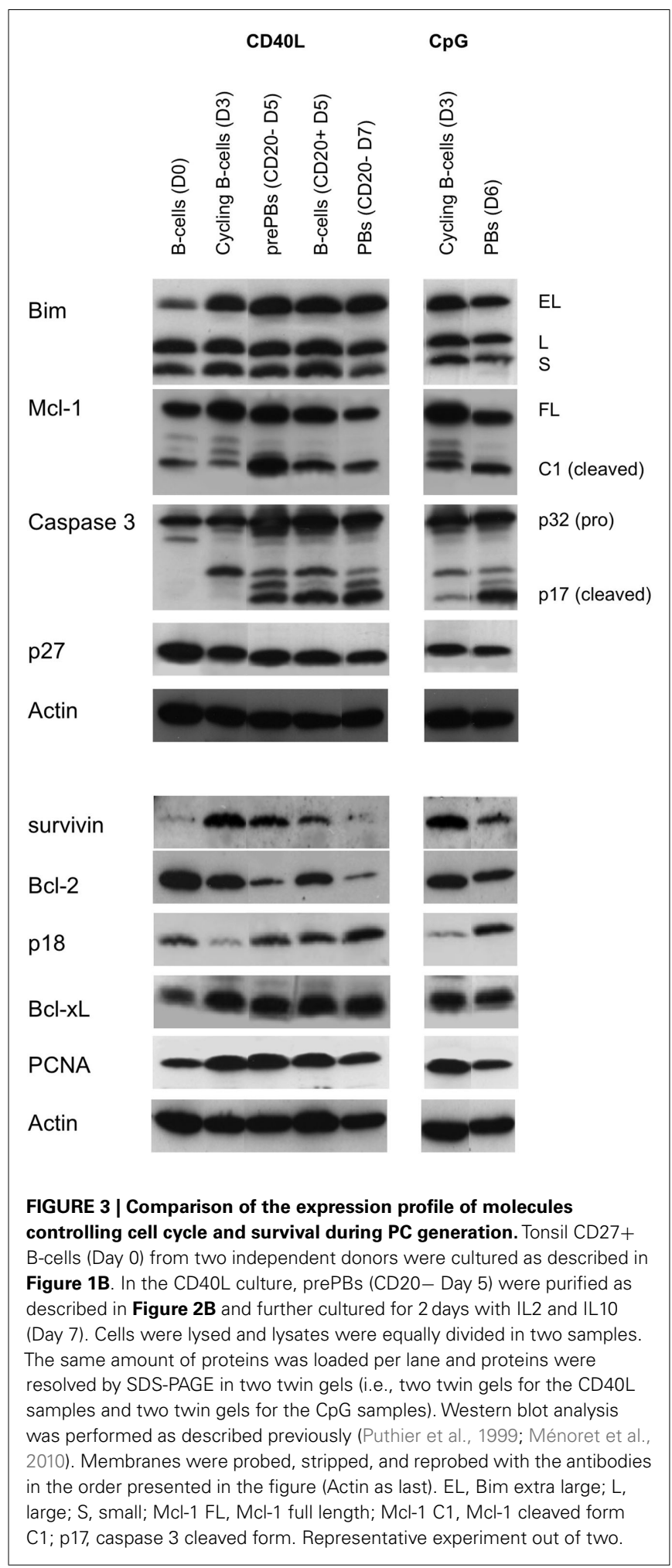

cultures. Compared with the IL2/IL10/CD40L culture, the CpG ODN/IL15 culture showed increased secretion of IgA (1.3- to 1.4fold-increase), $\operatorname{IgG}$ (1.45- to 1.56-fold-increase) and, importantly, $\operatorname{IgM}(2.02-7.22)$, as shown in Figure 4A. To assess the effect of CpG ODN on the same prePBs, we cultured IL2/IL10/CD40L-generated

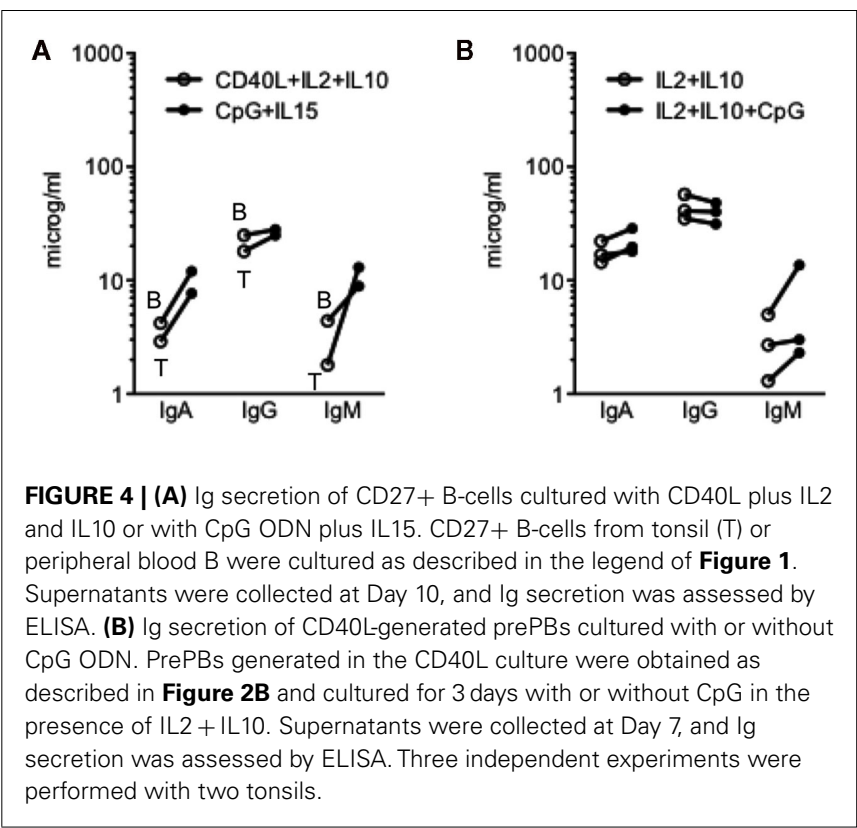

prePBs (Day 5) with or without CpG ODN for 2 days. As shown in Figure 4B, addition of CpG ODN significantly altered Ig secretion by increasing the secretion of IgM (median value 177\%) and IgA (median value 129\%) while decreasing that of $\operatorname{IgG}$ (median value $90 \%), p=0.0176$ ANOVA test. These data collectively indicate that IgM secretion was increased in the presence of $\mathrm{CpG}$ ODN, suggesting that $\mathrm{CpG}$ ODNs were more able than $\mathrm{CD} 40 \mathrm{~L}$ to induce the generation of IgM-secreting PCs.

CpG ODN DID NOT INDUCE CD20 EXPRESSION IN MYELOMA CELL LINES In humans, the archetype of malignant PCs is MM. MM is marked by the presence of a malignant clone secreting a monoclonal protein, often IgA or IgG and, very rarely, IgM (Kyle and Rajkumar, 2008). Myeloma cells are thought to be the malignant counterpart of bone marrow PCs. Similar to bone marrow PCs, MM cells are mainly CD20- (Robillard et al., 2003; Bataille et al., 2006). MM cells have been shown to express TLR9 and to respond to CpG ODN by increased proliferation and survival (Bohnhorst et al., 2006; Jego et al., 2006). In chronic lymphoid leukemia, a CD20+ neoplasm, CpG ODN was shown to increase the level of CD20 expression after 24-48 h (Mankaï et al., 2009). To assess whether CpG ODN could induce or increase CD20 expression in myeloma cells, eight TLR9+CD20- HMCLs, BCN, JIM3, MDN, NCI-H929, RPMI8226, SBN, U266 and XG6, and one TLR9+CD20+ HMCL, Karpas 620, were cultured for 2 days with $5 \mu \mathrm{g} / \mathrm{ml} \mathrm{CpG} \mathrm{ODN} 2006$. As shown in Figure 5, CpG ODN neither induced CD20 expression in CD20-TLR9+ HMCLs nor increased it in the CD20+TLR9+ HMCL. These data show that CpG ODN did not modulate CD20 expression in malignant IgG or IgA PCs.

\section{DISCUSSION}

In this paper, we show that $\mathrm{CpG}$ ODN and CD40L induce $\mathrm{PBs}$ and PCs with different features. Indeed, while CD40L promotes the generation of "classical" CD20-CD138- PBs and $\mathrm{CD} 20-\mathrm{CD} 138+\mathrm{PCs}, \mathrm{CpG}$ ODN promotes that of 


\section{CD20- HMCLs}

control
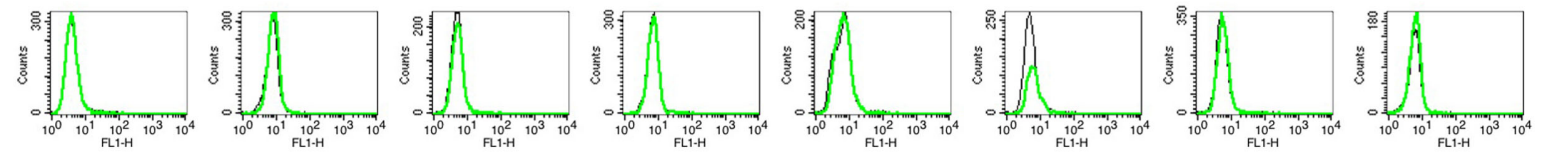

CpG
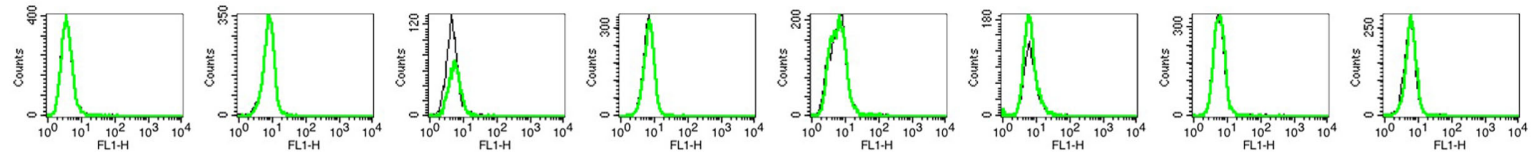

BCN

JIM3

\section{MDN}

\section{RPMI8226}

U266

XG6

\section{CD20+ HMCL}

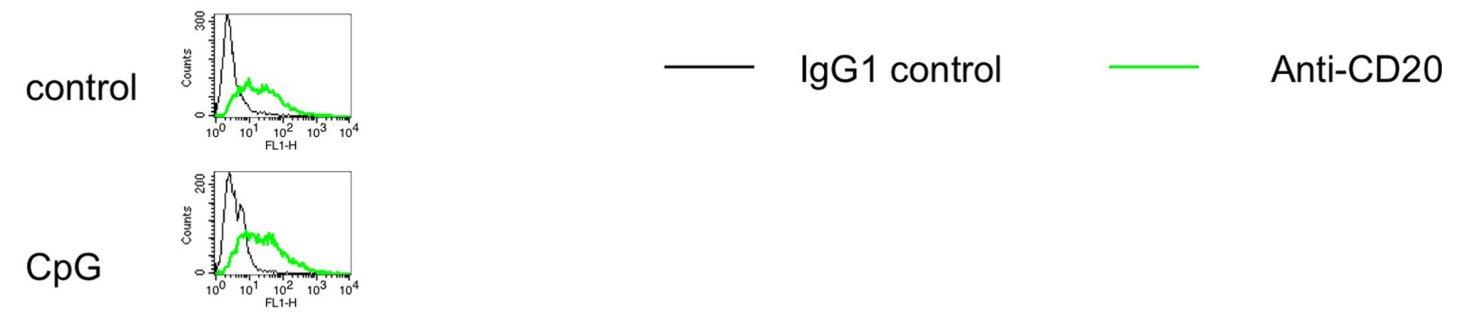

Karpas620

FIGURE 5 | CpG ODN neither induce nor increase CD20 expression on myeloma cell lines. Nine multiple myeloma cell lines expressing TLR9 were cultured for 2 days with or without CpG2006, and CD20 expression was analyzed by flow cytometry. Overlay histograms represent the CD20 staining over the control staining.

CD20+CD38+CD138+/- PBs/PCs and CD20-CD38+CD138+ PCs. This CD20+CD38+CD138+/- phenotype, which is not reminiscent of any B to PC intermediates (Pellat-Deceunynck et al., 1994; Arpin et al., 1997; Jego et al., 1999, 2001; Medina et al., 2002; Odendahl et al., 2005; Robillard et al., 2005; GeffroyLuseau et al., 2008; Jourdan et al., 2011), rather looks like to that of tonsil PCs (Medina et al., 2002; Robillard et al., 2005). It seems unlikely that CD20+ PCs could have emerged from CD27- cells because they represented a very weak contaminating population and because CpG ODN are not able to induce PC generation from CD27- B-cells unless anti-Ig are added (Bernasconi et al., 2002). The addition of CpG ODN to CD40L-induced prePBs increased the proportion of $\mathrm{CD} 20+\mathrm{PBs}$ and modified the Ig secretion isotype. It seems unlikely that CpG ODN simply increased CD20 expression because PBs obtained after the addition of CpG ODN were mainly $\mathrm{CD} 20-$. Our results collectively suggest that $\mathrm{CpG}$ ODN, which induced the proliferation of CD27+ memory Bcells, increased the survival of either CD20+ prePBs/PBs or both. Reciprocally, the removal of CpG ODN from CpG ODN induced prePBs decreased CD20 expression on PBs. This later result further suggests that CD20+ PBs have impaired survival without TLR9 stimulation. Analysis of proliferation and expression of proteins involved in proliferation, such as PCNA, did not demonstrate significant global differences between the two types of stimulation (Figures 1 and 3), arguing against a role of $\mathrm{CpG}$ in proliferation of CD20+CD38+CD138+/- cells. In the CD40L culture, CD40L was removed after 3 days because CD40L is known to block PC generation by inducing continuous $\mathrm{B}$ proliferation (Arpin et al., 1995). Interestingly, in contrast to CD40L, CpG ODN induced the proliferation of B-cells but did not block their subsequent differentiation. Because CpG ODN did not maintain proliferation, and its addition or removal subsequently impacted CD20 expression, we might conclude that it favored the survival of some CD20+ prePBs or CD20+ PBs. Jourdan et al. (2009) reported that, although IgM+ activated B-cells represented the major population of cycling Bcells after transient culture with soluble CD40L and CpG ODN, $\mathrm{IgM}+\mathrm{PBs}$ and $\mathrm{PCs}$ represented only a minor population, suggesting that either IgM PBs did not survive, in contrast to IgG PBs, or that IgM cycling B-cells were unable to differentiate into PBs in the absence of $\mathrm{CpG}$ ODN. Addition of CpG ODN to CD40L prePBs modified the Ig secretion (increase of IgM). Our data are in agreement with those of Bernasconi et al. (2002) who reported that CpG ODN and CD40L do not equally activate non-switched and switched CD27+ B-cells: IgM+ CD27+ B-cells efficiently respond to $\mathrm{CpG}$ ODN but not to CD40L in contrast to switched CD27+ B-cells, which do equally respond to both stimulations.

In malignant and normal B-cells, CpG ODN increase CD20 expression at both protein and mRNA levels but the mechanism(s) are not known (Jahrsdörfer et al., 2001; Mankaï et al., 2009). The effect of CpG ODN is more difficult to analyze in our study because cells differentiate and loose their CD20 phenotype during this process. Thus, CpG ODN either stimulate a subpopulation 
of CD27+ B-cells (IgM+?) committed to become CD20+ PBs and PCs or maintain CD20 expression on the PBs and PCs, which loose CD20 expression during their generation. Further experiments performed with cells isolated along all the differentiation process from pure switched and non-switched CD27+ B-cells are required to reach a definitive conclusion concerning the precise role of $\mathrm{CpG}$ ODN.

In vivo, $\mathrm{CD} 20+\mathrm{PBs}$ and $\mathrm{PCs}$ are found in tonsils, not within the bone marrow (Medina et al., 2002). PCs from tonsils express a low level of CD138 in contrast to PCs from bone marrow (Medina et al., 2002; Robillard et al., 2005). Thus, based on the phenotype, CD20+CD138+/- cells obtained with CpG ODN look like to tonsil PCs. In humans, PCs are also found in the lamina propria, in spleen and lymph nodes, and in the rheumatoid synovium (Dechanet et al., 1995; Medina et al., 2002; Moser et al., 2006; Radbruch et al., 2006). In humans, the phenotype of PCs has been mainly studied in tonsils and bone marrow, not in spleen or lymph nodes. To date, only tonsil PCs have been reported to express CD20. The CD20+CD38+CD138+/- phenotype of tonsil PCs does not correlate with an intermediate stage of differentiation because CD20 expression is lost before CD138 appearance (Jego et al., 1999, 2001; Odendahl et al., 2005). Thus, there is to date no obvious explanation for the particular phenotype of tonsil PCs. Because, tonsillectomies are performed in children and teenagers with recurrent bacterial infections of tonsils, it is possible that the CD20+ phenotype of PCs might be related to the recurrent infections rather than to the tonsilar location. Further analysis of phenotype in various lymphoid organs (tonsil, spleen, lymph node) in the absence of recurrent bacterial infections could determine whether this $\mathrm{CD} 20+$ phenotype is related to infections or to location, or both.

Although TLRs are widely expressed on malignant B-cells, there is no evidence to date that chronic activation of B-cells is involved in the emergence or growth or survival of malignant Bcells in vivo. MM and WM (Waldenstrom macroglobulinemia) are two PC malignancies characterized by a monoclonal peak of immunoglobulin of $\operatorname{IgG}$, (or IgA), or IgM isotype, respectively;

\section{REFERENCES}

Arpin, C., Banchereau, J., and Liu, Y. J. (1997) Memory B cells are biased towards terminal differentiation: a strategy that may prevent repertoire freezing. J. Exp. Med. 186, 931-940.

Arpin, C., Déchanet, J., Van Kooten, C., Merville, P., Grouard, G., Brière, F., Banchereau, J., and Liu, Y. J. (1995). Generation of memory B cells and plasma cells in vitro. Science 268, 720-722.

Banwait, R., O’Regan, K., Campigotto, F., Harris, B., Yarar, D., Bagshaw, M., Leleu, X., Leduc, R., Ramaiya, N., Weller, E., and Ghobrial, I. M. (2011). The role of $18 \mathrm{~F}-\mathrm{FDG}$ PET/CT imaging in Waldenstrom macroglobulinemia. Am. J. Hematol. 86, 567-572.

Bataille, R., Jégo, G., Robillard, N., Barillé-Nion, S., Harousseau, J.

however, in contrast to MM cells that are mainly located within the bone marrow, WM cells are mainly found within the spleen and lymph nodes (Chng et al., 2006; Kyle and Rajkumar, 2008; Banwait et al., 2011). MM cells exhibit all the hallmarks of Ag-selected switched B-cells, while WM cells exhibit those of Ag-selected nonswitched B-cells (Martín-Jiménez et al., 2007). In contrast to MM cells, WM cells are a mix of lymphoid (CD19+ CD20+ CD138-) and lymphoplasmacytic cells (CD19+ CD20+ CD138+/-; Chng et al., 2006; Hodge et al., 2011). It would be of particular interest to establish and compare the gene expression profiles of WM cells, tonsil PCs and CpG ODN-derived CD20+ PBs/PCs.

Plasmablasts and PCs, which are highly susceptible to apoptosis, are primed for death by the expression of BH3-only molecules of the Bcl-2 family (Jego et al., 1999; Vikstrom and Tarlinton, 2011). Only interactions with the local microenvironment, which might include osteoclasts, can rescue them from death, explaining the long-life of resident bone marrow PCs (Odendahl et al., 2005; Moser et al., 2006; Geffroy-Luseau et al., 2008). In vitro, the generation of PBs and PCs from B-cells is always marked by a high incidence of apoptosis, as shown by the high incidence of cells in subG1 peak (Figure 2D and Jego et al., 1999, 2001). In this study, we show that apoptosis may be explained by the cleavage of Mcl-1 and the activation of caspase 3. Activation of B-cells induces an increase of Mcl-1, Bcl-xL, and Bim and a decrease of Bcl-2, suggesting that $\mathrm{PBs}$ and $\mathrm{PCs}$ should be rather Mcl- 1 or Bcl-xL primed for death while resting memory B-cells should be rather Bcl-2 primed for death (Figure 3 and Vikstrom and Tarlinton, 2011). The modulation of expression of these molecules was very similar in both CD40L and CpG cultures, showing that it is an intrinsic feature of PBs and PCs. In summary, we have shown that CpG ODN induced the in vitro generation of $\mathrm{CD} 20+\mathrm{PBs}$ and $\mathrm{CD} 20+$ PCs from CD27+ memory B-cells.

\section{ACKNOWLEDGMENTS}

Supported by the Ligue Nationale Contre Le Cancer (Equipe labellisée 2008). The authors thank Pr. R. Bataille and Dr. N. Robillard for their critical review of the manuscript.

and tumorigenesis. Immunol. Rev. 194, 39-47.

Chiron, D., Pellat-Deceunynck, C., Maillasson, M., Bataille, R., and Jego, G. (2009). Phosphorothioatemodified TLR9 ligands protect cancer cells against TRAILinduced apoptosis. J. Immunol. 183, 4371-4377.

Chng, W. J., Schop, R. F., Price-Troska, T., Ghobrial, I., Kay, N., Jelinek, D. F., Gertz, M. A., Dispenzieri, A., Lacy, M., Kyle, R. A., Greipp, P. R., Tschumper, R. C., Fonseca, R., and Bergsagel, P. L. (2006). Geneexpression profiling of Waldenstrom macroglobulinemia reveals a phenotype more similar to chronic lymphocytic leukemia than multiple myeloma. Blood 108, 2755-2763.

De Vos, J., Hose, D., Reme, T., Tarte, K., Moreaux, J., Mahtouk, K., Jourdan,
M., Goldschmidt, H., Rossi, J. F., Cremer, F. W., and Klein, B. (2006). Microarray-based understanding of normal and malignant plasma cells. Immunol. Rev. 210, 86-104.

Dechanet, J., Merville, P., Durand, I., Banchereau, J., and Miossec, P. (1995). The ability of synoviocytes to support terminal differentiation of activated B cells may explain plasma cell accumulation in rheumatoid synovium. J. Clin. Invest. 95, 456-463.

Geffroy-Luseau, A., Jégo, G., Bataille, R., Campion, L., and PellatDeceunynck, C. (2008). Osteoclasts support the survival of human plasma cells in vitro. Int. Immunol. 20, 775-782.

Hodge, L. S., Novak, A. J., Grote, D. M., Braggio, E., Ketterling, R. P., Manske, M. K., Price Troska, T. L., 
Ziesmer, S. C., Fonseca, R., Witzig, T. E., Morice, W. G., Gertz, M. A., and Ansell, S. M. (2011). Establishment and characterization of a novel Waldenstrom macroglobulinemia cell line, MWCL-1. Blood 117, e190-197.

Jahrsdörfer, B., Hartmann, G., Racila, E., Jackson, W., Mühlenhoff, L., Meinhardt, G., Endres, S., Link, B. K., Krieg, A. M., and Weiner, G. J. (2001). CpG DNA increases primary malignant $\mathrm{B}$ cell expression of costimulatory molecules and target antigens. J. Leukoc. Biol. 69, 81-88.

Jego, G., Bataille, R., Geffroy-Luseau, A., Descamps, G., and PellatDeceunynck, C. (2006). Pathogenassociated molecular patterns are growth and survival factors for human myeloma cells through toll-like receptors. Leukemia 20, 1130-1137.

Jego, G., Bataille, R., and PellatDeceunynck, C. (2001). Interleukin6 is a growth factor for nonmalignant human plasmablasts. Blood 97, 1817-1822.

Jego, G., Robillard, N., Puthier, D., Amiot, M., Accard, F., Pineau, D., Harousseau, J. L., Bataille, R., and Pellat-Deceunynck, C. (1999). Reactive plasmacytoses are expansions of plasmablasts retaining the capacity to differentiate into plasma cells. Blood 94, 701-712.

Jourdan, M., Caraux, A., Caron, G., Robert, N., Fiol, G., Rème, T., Bolloré, K., Vendrell, J. P., Le Gallou, S., Mourcin, F., De Vos, J., Kassambara, A., Duperray, C., Hose, D., Fest, T., Tarte, K., and Klein, B. (2011). Characterization of a transitional preplasmablast population in the process of human B cell to plasma cell differentiation. J. Immunol. 187, 3931-3941.

Jourdan, M., Caraux, A., De Vos, J., Fiol, G., Larroque, M., Cognot, C.,
Bret, C., Duperray, C., Hose, D., and Klein, B. (2009). An in vitro model of differentiation of memory B cells into plasmablasts and plasma cells including detailed phenotypic and molecular characterization. Blood 114, 5173-5181.

Kyle, R. A., and Rajkumar, S. V. (2008). Multiple myeloma. Blood 111, 2962-2972.

Mankaï, A., Buhé, V., Hammadi, M., Youinou, P., Ghedira, I., Berthou, C., and Bordron, A. (2009). Improvement of rituximab efficiency in chronic lymphocytic leukemia by CpG-mediated upregulation of CD20 expression independently of PU.1. Ann. N. Y. Acad. Sci. 1173, 721-728.

Martín-Jiménez, P., García-Sanz, R., Balanzategui, A., Alcoceba, M., Ocio, E., Sanchez, M. L., González, M., and San Miguel, J. (2007). Molecular characterization of heavy chain immunoglobulin gene rearrangements in Waldenström's macroglobulinemia and IgM monoclonal gammopathy of undetermined significance. Haematologica 92, 635-642.

Medina, F., Segundo, C., Campos-Caro, A., Gonzalez-Garcia, I., and Brieva, J. A. (2002). The heterogeneity shown by human plasma cells from tonsil, blood, and bone marrow reveals graded stages of increasing maturity, but local profiles of adhesion molecule expression. Blood 99, 2154-2161.

Ménoret, E., Gomez-Bougie, P., Surget, S., Trichet, V., Oliver, L., Pellat-Deceunynck, C., and Amiot, M. (2010). Mcl-1128-350 fragment induces apoptosis through direct interaction with Bax. FEBS Lett. 584, 487-492.

Moreaux, J., Klein, B., Bataille, R., Descamps, G., Maïga, S., Hose, D., Goldschmidt, H., Jauch, A., Rème, T.,
Jourdan, M., Amiot, M., and PellatDeceunynck, C. (2011). A high-risk signature for patients with multiple myeloma established from the molecular classification of human myeloma cell lines. Haematologica 96, 574-582.

Moser, K., Tokoyoda, K., Radbruch, A. MacLennan, I., and Manz, R. A. (2006). Stromal niches, plasma cell differentiation and survival. Curr. Opin. Immunol. 18, 265-270.

Odendahl, M., Mei, H., Hoyer, B. F. Jacobi, A. M., Hansen, A., Muehlinghaus, G., Berek, C., Hiepe, F., Manz, R., Radbruch, A., and Dörner, T. (2005). Generation of migratory antigen-specific plasma blasts and mobilization of resident plasma cells in a secondary immune response. Blood 105, 1614-1621.

Pellat-Deceunynck, C., Bataille, R. Robillard, N., Harousseau, J. L., Rapp, M. J., Juge-Morineau, N., Wijdenes, J., and Amiot, M. (1994) Expression of CD28 and CD40 in human myeloma cells: a comparative study with normal plasma cells. Blood 84, 2597-2603.

Puthier, D., Pellat-Deceunynck, C., Barillé, S., Robillard, N., Rapp, M. J., Juge-Morineau, N., Harousseau, J. L., Bataille, R., and Amiot, M. (1999). Differential expression of Bcl-2 in human plasma cell disorders according to proliferation status and malignancy. Leukemia 13, 289-294.

Radbruch, A., Muehlinghaus, G., Luger, E. O., Inamine, A., Smith, K. G., Dorner, T., and Hiepe, F. (2006). Competence and competition: the challenge of becoming a long-lived plasma cell. Nat. Rev. Immunol. 6, 741-750.

Robillard, N., Avet-Loiseau, H., Garand, R., Moreau, P., Pineau, D., Rapp, M. J., Harousseau, J. L., and
Bataille, R. (2003). CD20 is associated with a small mature plasma cell morphology and $t(11 ; 14)$ in multiple myeloma. Blood 103, 1070-1071.

Robillard, N., Pellat-Deceunynck, C., and Bataille, R. (2005). Phenotypic characterization of the human myeloma cell growth fraction. Blood 105, 4845-4848.

Shapiro-Shelef, M., and Calame, K. (2005). Regulation of plasma-cell development. Nat. Rev. Immunol. 5, 230-242.

Vikstrom, I., and Tarlinton, D. M. (2011). B cell memory and the role of apoptosis in its formation. Mol. Immunol. 48, 1301-1306.

Conflict of Interest Statement: The authors declare that the research was conducted in the absence of any commercial or financial relationships that could be construed as a potential conflict of interest.

Received: 14 October 2011; accepted: 11 December 2011; published online: 30 December 2011.

Citation: Geffroy-Luseau A, Chiron D, Descamps $G$, Jégo $G$, Amiot $M$ and Pellat-Deceunynck C (2011) TLR9 ligand induces the generation of CD20+ plasmablasts and plasma cells from CD27+ memory B-cells. Front. Immun. 2:83. doi: 10.3389/fimmu.2011.00083

This article was submitted to Frontiers in B Cell Biology, a specialty of Frontiers in Immunology.

Copyright (C) 2011 Geffroy-Luseau, Chiron, Descamps, Jégo, Amiot and PellatDeceunynck. This is an open-access article distributed under the terms of the Creative Commons Attribution Non Commercial License, which permits noncommercial use, distribution, and reproduction in other forums, provided the original authors and source are credited. 